\title{
The surgeon
}

\author{
Domingo BRAILE
}

Physician has always existed and he will exist forever! In the most primitive societies, there he was to pursue his art, resulting from an inner man impulse, independent of any interest or will. We can mention by an instance, without the interest of being unabridged the following: medicinemen, witch-doctors, shamans, quack, quack doctors, praying men, sorceresses, midwives, and Barber-Surgeons. The latter ones are the ancient repository of knowledge that over the years eventually create a major medical specialty within the Medicine. It keeps with the Medicine close relations, but it depends of the unrestrictedly unique skills of the practitioners.

The Barber-Surgeon took with himself his clumsy working tools, existent in those days and his own hospital, usually a carriage drawn by horses. He wandered from one town to another offering his services to those who suffered from incurable diseases through the noninvasive medicine from those times.

Masters who taught their disciple the techniques they have developed or learnt throughout their lives passed down their knowledge from one generation to the next. The diseases that could be treated surgically were few. They were always followed by great risk and suffering, because infections and bleedings were a constant, and anesthesia was still just a dream.

The Barber-Surgeons were not always successful in their procedures and the death of patients was not a rare event. When such an event occurred, they ran away in the dead of the night before the families and friends of the deceased massacred them. One of the prerequisites to get rid of the death was their Ambulant Hospitals drawn by horses: They had to be very strong and fast so that they could have a well-succeed escape!

Through evolution, these men endowed with innate qualities, which allowed them to penetrate the "insides" of their fellow creatures, have been accepted at medical schools. They became part of the elite of those who dedicated themselves to master the knowledge of anatomy, physiology, and pathology. These major frameworks would transform the empirical medicine into science. However, the art continued to be a routine for surgeons. It does not matter how much he holds the whole field of sciences, if he does not have the necessary skills, he at no time will be succeeded.

The advent of general anesthesia, which took place on October 16, 1846, by the insistence of the dentist William Thomas Green Morton, allowed the surgeon John Collins Warren, from the Massachusetts General Hospital, in Boston, to remove a tumor from the neck of Gilbert Abbot, a 17-year-old young boy. He was seemingly unconscious during the entire procedure without feeling any pain.

The field of modern surgery as we know it today has been opened. Over this short span of only 160 years, almost nothing in the millenary evolution of humanity, the surgery has reached limits never imagined before. Endotracheal intubation, an amazingly simple concept but so difficult to be accepted, has allowed the physicians to open the chests of the patients and make anesthesia safer and reproducible.

The possibility of open heart surgeries, with cardiopulmonary bypass developed by John Gibbon in 1953, was a conclusive landmark of surgery as a science. The latest possibility of performing video-assisted thoracic surgeries with the ability to make smaller incision progress rapidly to turn the surgeon less and less aggressive and the patient more tolerant of interventions. There is an old aphorism that should never be forgotten: the body does not allow large invasions and reacts to them with the systemic inflammatory response syndrome (SIRS), it is often life threatening, or difficult to control. Therefore, it is clear the importance of the minimally invasive surgeries, a dramatic breakthrough in surgery.

All this progress was achieved with great difficulty and considerable investments in research and development of equipment, which are getting more and more complex technologically. It became imperative to build Hybrid Operating Rooms, where the surgeon can exert his activity with the aid of all the necessary technology.

These hybrid operating rooms are something like operating theatres of appropriate dimensions, where it is possible to have high-resolution image devices, video fluoroscopy and videoscopy with lighting systems, ultrasound, and single or three-dimensional 
echocardiography, as well as advanced equipment for online monitoring of all vital constants.

It is essential to have reliable and automated anesthesia systems, as well as all life-support devices, such as cardiopulmonary bypass pumps and oxygenators, and modalities of extracorporeal circulation, as "extracorporeal membrane oxygenation" (ECMO) and "extracorporeal lung assist" (ECLA). Surgeons will be able carried out their operations with some of these devices without being limited by illumination and visibility of all vessels, organs, and structures of the human body. This way they can choose the most suitable procedures according to the patient needs.

The surgeon will be able to use a conclusive endovascular intervention, complementing it with a minimally invasive surgery, or even converting it into an open surgery, offering maximum safety and positive outcome for the patient, who has placed all his confidence in him.
As a result, the multidisciplinary health team should have more professional betterment and be well versed in the pure sciences, to work with the wide range of devices existing in a modern hospital. These devices represent the expression of the technological breakthrough that permeated in an outstanding way the twentieth century and millennium in which we live in.

The future of surgery involves the incorporation of techniques farther more advanced, proceeding from the most varied fields of scientific research, since the creation of the CCD (Charge-coupled device, which we have already seen in any digital camera) to the remarkable achievements necessary to make a space flight a realistic possibility.

*Editor in Chief of the Brazilian Journal of Cardiovascular Surgery 